\title{
Distortion Analysis Toolkit-A Software Tool for Easy Analysis of Nonlinear Audio Systems
}

\author{
Jyri Pakarinen \\ Department of Signal Processing and Acoustics, School of Science and Technology, Aalto University, \\ P.O. Box 13000, 00076 Aalto, Finland \\ Correspondence should be addressed to Jyri Pakarinen, jyri.pakarinen@tkk.fi
}

Received 27 February 2010; Revised 30 April 2010; Accepted 17 May 2010

Academic Editor: Udo Zoelzer

Copyright ( 2010 Jyri Pakarinen. This is an open access article distributed under the Creative Commons Attribution License, which permits unrestricted use, distribution, and reproduction in any medium, provided the original work is properly cited.

Several audio effects devices deliberately add nonlinear distortion to the processed signal in order to create a desired sound. When creating virtual analog models of nonlinearly distorting devices, it would be very useful to carefully analyze the type of distortion, so that the model could be made as realistic as possible. While traditional system analysis tools such as the frequency response give detailed information on the operation of linear and time-invariant systems, they are less useful for analyzing nonlinear devices. Furthermore, although there do exist separate algorithms for nonlinear distortion analysis, there is currently no unified, easy-touse tool for rapid analysis of distorting audio systems. This paper offers a remedy by introducing a new software tool for easy analysis of distorting effects. A comparison between a well-known guitar tube amplifier and two commercial software simulations is presented as a case study. This freely available software is written in Matlab language, but the analysis tool can also run as a standalone program, so the user does not need to have Matlab installed in order to perform the analysis.

\section{Introduction}

Since the 1990s, there has been a strong trend in the digital audio effects community towards virtual analog modeling, that is, mimicking the sound of old analog audio devices using digital signal processing (DSP). Guitar tube amplifier emulation [1] has been an especially vibrant area of research with several commercial products.

There are roughly two methodological approaches for designing virtual analog models: the black-box- and the white-box methods. In the former, the designer treats the original audio device (called target in the following) as an unknown system and tries to design the model so that it mimics only the input/output relationship of the target, without trying to simulate its internal state. In the white-box approach, the designer first studies the operation logic of the device (often from the circuit schematics) and tries to design the model so that it also simulates the internal operation of the target. While both approaches can yield satisfactory results in many cases, there is still room for improvement in the sound quality and model adjustability, if the virtual analog models are to act as substitutes for the original analog devices.

In all virtual analog modeling, the target and model responses need to be carefully analyzed. This is obviously essential in the black-box approach, but it is also an unavoidable step in white-box modeling, since even circuit simulation systems need to have their component values adjusted so that the model response imitates the target response. While some part of this analysis can be conducted simply by listening to the models, there is a clear need for objective analysis methods. If the target system can be considered linear and time invariant (LTI), it is relatively straightforward to analyze the magnitude and phase responses of the target and model and try match them as closely as possible. For distorting systems, however, linear magnitude and phase responses give only partial information on how the system treats audio signals, since distortion components are not analyzed.

Analysis of mildly distorting audio systems, such as loudspeakers, have traditionally consisted of simple total harmonic distortion (THD) measurements, where a static 
sine signal is fed to the system as an input, and the harmonic distortion component levels are summed and normalized to the amplitude of the fundamental component. Even though THD gives a vague indication on the general amount of static harmonic distortion, it tells nothing on the type of this distortion. Thus, THD is a poor estimate for analyzing distorting audio effects. Although more informative analysis techniques have been designed by the scientific community, there is currently no unified, easy-to-use tool for simultaneously conducting several distortion analysis measurements.

\section{Distortion Analysis Toolkit}

2.1. Overview. The distortion analysis toolkit (DATK) is a software tool for analyzing the distortion behavior of real and virtual audio effects. It operates by first creating a userdefined excitation signal and then analyzes the responses from the devices. The DATK package includes five analysis techniques, which can be further augmented with additional user-defined analysis functions. The DATK is developed in Matlab language, but standalone operation is also possible. Since a distortion measurement on an audio effects device usually consists of making several individual recordings while varying some control parameter and keeping the other parameters fixed, the DATK is designed for batch processing. This means that the DATK creates an excitation signal as an audio file according to the specifications set by the user. Next, the user plays this excitation to the device under test (DUT) and records the output on any playback/recording software. The DUT response to its control parameter variations can be tested by changing the control parameter values and rerecording the response. As a result, the user is left with a set of response files that each have different control parameter settings.

The analysis part is carried out by pointing the excitation file and the set of response files to the DATK, after which it plots analysis figures, so that each analysis type is shown as a separate figure, and the analysis results from each response file are illustrated with subfigures. Thus, it is easy to interpret the effect of control parameter variations on the system response, since each subfigure shows the response to a different value of the varied parameter. The analysis figures can also be saved as.fig files even in standalone mode, allowing further editing with Matlab. The DATK runs on any modern computer, and only requires the use of a simple playback/recording software for playing the excitation signal and recording the responses. For measuring software plugins, the playback/recording software should be able to act as a plugin host. For measuring physical effects devices, an audio interface with adjustable playback/recording gains should be used. Sections 2.2 through 2.6 discuss the analysis functions currently included in the DATK, while Section 2.7 discusses how to develop additional analysis functions. Since the user defines the desired analysis techniques and their parameters by writing a text file, where the different analysis types (or same analysis types with different parameters) are written as separate lines, Sections 2.2 through 2.6 also introduce the syntax for performing each analysis type. The actual use of the DATK software is illustrated in Section 3 with a case study.

2.2. Sine Analysis. One of the simplest distortion analysis techniques is to insert a single sinusoid signal with fixed amplitude and frequency into the system, and plot the resulting spectrum. The advantage of this analysis type is that it is easy to understand and the results are straightforward to interpret. The disadvantage is that this analysis gives only information on how the system treats a single, static sine signal with given amplitude and frequency. In particular, it tells nothing about the dynamic behavior of the system. The DATK sine analysis function is invoked using the syntax

$$
\text { SineAnalysis (frequency, duration) }
$$

where the frequency denotes the frequency of the analysis sine in Hertz, and duration is the signal duration in seconds. The amplitude of the sine is set to unity. In the analysis phase, the DATK plots the resulting system response spectrum in the audio frequency range so that the amplitude of the fundamental frequency component (i.e., the component at the same frequency as the analysis sine) is $0 \mathrm{~dB}$. The fundamental component is further denoted with a small circle around its amplitude peak, which helps in locating it if the spectrum has high-amplitude distortion components at subharmonic- or otherwise low frequencies.

2.3. Logsweep Analysis. The logsweep analysis technique is an ingenious device for analyzing static harmonic distortion as a function of frequency. Introduced by Farina in 2000 [2], the basic idea behind the logsweep analysis technique is to insert a logarithmic sine sweep signal with fixed amplitude to the system, and then convolve the time-reversed and amplitude weighted excitation signal with the system response. As a result, one obtains an impulse response signal, where the response of each harmonic distortion component is separated in time from the linear response and each other. If the duration of the logsweep signal is long enough, the time separation between the harmonic impulse responses is clear and it is straightforward to cut the individual harmonic responses and represent them in the frequency domain. As a result, one obtains magnitude response plots for the linear response and each harmonic distortion component.

The DATK logsweep analysis function uses the syntax

$$
\begin{aligned}
& \text { LogsweepAnalysis (start_freq, end_freq, } \\
& \text { duration, number_of_harmonics) }
\end{aligned}
$$

where start_freq and end_freq are the frequency limits (in $\mathrm{Hz}$ ) for the logarithmic sweep, and duration is the sweep duration in seconds. The parameter number_of harmonics sets the number of harmonic components to plot in the analysis phase, so that value 3 , for example, would correspond to the linear response and the 2nd and 3rd harmonic distortion components to be drawn. As a rule of thumb, the signal to noise ratio (SNR) decreases with increasing harmonic numbers, so it is often not advisable to try to analyze a large number of harmonics 
(e.g., >6), unless long excitation signals (several seconds) are used.

In the analysis phase, the DATK plots the linear and harmonic distortion component magnitude responses as a function of frequency (in the frequency range from start_freq to end_freq) in a single figure. The response curves are illustrated with different colors and each curve has a number denoting the harmonic order, starting from 1 for the linear response, 2 for the 2 nd harmonic, and so forth. The magnitude offset of the responses is set so that the magnitude of the linear response is $0 \mathrm{~dB}$ at low frequencies. The magnitude responses of the harmonic distortion components are shifted down in frequency by the order of their harmonic number. This means that looking at the analysis figure at a given frequency, say at $1 \mathrm{kHz}$, curve 1 gives the magnitude of the linear component, curve 2 gives the magnitude of the 2nd harmonic (which actually has the frequency of $2 \mathrm{kHz}$ ), curve 3 gives the magnitude of the $3 \mathrm{rd}$ harmonic (residing at $3 \mathrm{kHz}$ ), and so forth. This will be further illustrated in Section 3.6. The harmonic impulse responses are windowed using a Blackman window prior to moving them into the frequency domain, for obtaining smoother magnitude response plots [3].

The advantage of this measurement technique over the sine analysis (Section 2.2) is that it displays more information in a single figure by drawing the magnitude responses as a function of the (fundamental) frequency on the full audio frequency range. For analyzing the magnitude of high-order distortion components, however, the sine analysis is usually better since it displays the entire audible spectrum in one figure, while the logsweep analysis can successfully be used to track only the low-order distortion components, due to clarity reasons and the aforementioned SNR issue. Another drawback of the logsweep analysis technique is that long excitation signals can take a long time to analyze due to the relatively slow computation of long convolutions.

2.4. Intermodulation Distortion Analysis. When a multitone signal is clipped, intermodulation distortion (IMD) occurs. This means that the distortion creates signal components not only to the integer multiples of the original tones, but also to frequencies which are the sums and differences of the original signal components and their harmonics. In musical context, IMD is often an undesired phenomenon, since IMD components generally fall at frequencies which are not in any simple harmonic relation to the original tones, making the resulting sound noisy and inharmonic.

Traditionally, the IMD of distorting systems has been measured by feeding a sum of two sinusoids with different frequencies into the system and plotting the resulting spectrum. Although this type of analysis gives an exact indication of the frequencies and amplitudes of the distortion components for a given pair of input signal frequencies and amplitudes, it is not very useful in determining the overall level of the IMD. It should be noted that with distorting audio effects, it is often useful to know the exact frequency and amplitude behavior of the harmonic distortion components, since there are some strong opinions regarding the harmonic content and the resulting sound, such as "the vacuum tube sound consists mainly of even harmonics", or that "the 7th harmonic is something to be avoided". With IMD, however, it is probably more useful to have a single number indicating the overall amount of distortion, since IMD is generally considered an undesired phenomenon, with no specific preferences on any particular IMD components. Furthermore, traditional IMD measurements ignore the dynamic behavior of the intermodulation mechanism, so that dynamic or transient intermodulation (DIM/TIM) [4] distortion components are left unnoticed.

The IMD analysis of the DATK software is based on the measurement technique introduced by Leinonen et al. [5], which measures both the DIM distortion and static IMD. In this measurement approach, a square wave signal with a fundamental frequency $f_{1}$ is summed with a loweramplitude sinusoid having a frequency $f_{2}$, so that $f_{1}<$ $f_{2}$. Next, this signal is inserted to the DUT, and the amplitude of the IMD components at audio frequency range is compared to the amplitude of the sinusoid. As a result, a percentage ratio between the root-mean-square (RMS) IMD components and the low-amplitude sinusoid riding on top of the square wave is obtained. Thus, an IMD percentage value of $100 \%$ would mean that the RMS IMD is equal in magnitude to the frequency component that causes the IMD in the first place. The static IMD is measured in a very similar way, the only difference being that instead of a square wave signal, a triangular waveform is used.

For measuring the IMD, the DATK creates two signals: one containing the square wave and the sine wave (for measuring DIM), and one containing the triangle wave and the sine (for measuring static IM). In order to estimate the IMD as a function of input signal amplitude, both of these signals are weighted by a linear amplitude ramp, so that the overall signal amplitude increases with time, although the amplitude ratios between the square (or triangle) wave and the sine wave remain unchanged. The DATK IMD analysis is called with the function

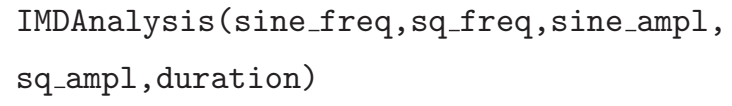

where sine_freq and sine_ampl are the frequency $(\mathrm{Hz})$ and amplitude of the sinusoid, respectively, and sq_freq and sq_ampl are the fundamental frequency and amplitude of the square wave. The triangle wave uses the same frequency and amplitude parameters as the square wave. The frequencies of the sine and square waves should be selected so that they are not in an integer relation with each other or the sampling frequency of the system.

The final parameter, duration, sets the length (in seconds) of each of the IMD test signals. The amplitude parameters are normalized so that the ratio between the sine and the square wave amplitudes remains sine_ampl/sq_ampl, but the overall signal amplitude reaches unity at the end of the test signal ramp. For avoiding digital aliasing of the square and triangle wave signals, the DATK creates them using the differentiated parabolic waveform technique, introduced in [6]. At the analysis phase, the DATK analyzes the system's 
output spectra for the IMD test signals and evaluates the DIM and IM percentages.

The advantage of the IMD measurement suggested in [5] over the traditional IMD measurements (i.e. simply plotting the output spectra), is that it provides two intuitive parameters concerning the IMD level: the IM percentage, which tells the amount of IMD associated with relatively static signals (such as the sound of a violin), and the DIM percentage, which tells the amount of IMD associated with transient signals (such as the sound of percussions).

2.5. Transient Response Analysis. Measuring the transient behavior of distorting audio devices is difficult in general. Typically, the transient response depends heavily on the amplitude and frequency content of the test signal, so it is usually not possible to generalize the results for other types of input signals. Nevertheless, it is still useful to compare the input/output waveforms of a distorting device when a transient signal is used as input, for example because certain types of dynamic distortion (such as the blocking distortion, as in [7]) can be identified from the waveforms.

The DATK transient response analysis operates by creating a sine burst signal, where the first cycle (called transient) has a larger amplitude than rest of the sine burst (called tail). The DATK transient response analysis function uses the syntax

$$
\begin{aligned}
& \text { TransientAnalysis (tail_ampl, freq, } \\
& \text { duration, cycles_to_draw) }
\end{aligned}
$$

where tail_ampl denotes the amplitude of the tail of the sine burst following the transient, and should be a positive number smaller than one (the amplitude of the transient is always unity). Parameters freq and duration set the frequency $(\mathrm{Hz})$ and duration $(\mathrm{sec})$ of the sine burst, respectively. The parameter cycles_to_draw sets the initial zoom limit in the analysis figure, so that dynamic distortion effects are easy to see. For example, a value of 10 would result in a waveform analysis figure, where 10 cycles of the response wave were displayed. The optimal value for the cycles_to_draw parameter obviously depends on the dynamic distortion characteristics of the device. If the DUT acts only as a static nonlinearity, the amplitude envelope, horizontal offset, and shape of the tail should remain constant in the analysis figure.

2.6. Aliasing Analysis. The DATK also has a tool for estimating the effect of signal aliasing in digital distorting devices. Nonlinear distortion always expands the signal spectrum by creating harmonic (and other) distortion components. This is problematic in a digital implementation, since the distortion components exceeding the Nyquist frequency (half the sampling rate) will alias back to the baseband, possibly resulting in an inharmonic and noisy signal. There are several techniques for avoiding aliasing within digital distortion [1], oversampling being probably the most popular.

The aliasing analysis tool in the DATK operates by creating a high-frequency sine signal, and analyzing the spectrum of the system response. This analysis technique, introduced

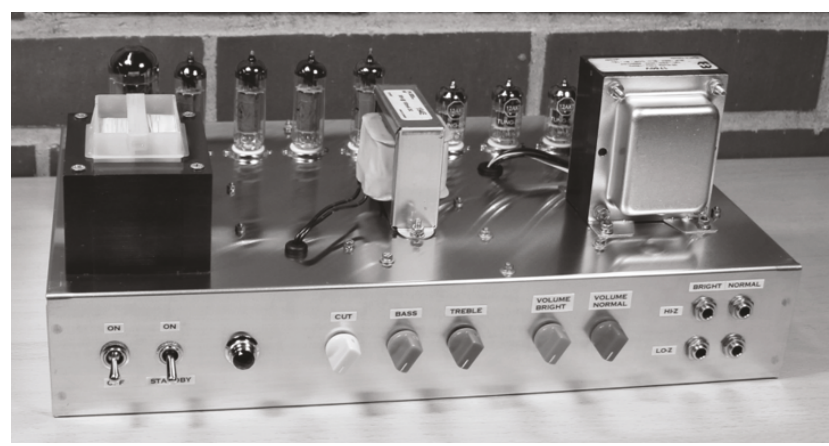

FIgure 1: The custom-built AC30 tube guitar amplifier (photo by Ari Viitala).

in a recent journal article [7], tracks the nonaliased harmonic distortion components and fits a simple auditory spectrum curve on them, for estimating the frequency masking effect of the baseband signal. More specifically, the auditory spectrum curve is estimated by fitting a gammatone filterbank [8] magnitude response curve on top of the nonaliased signal so that the center frequencies of the filters are aligned with the frequencies of the baseband components. A fixed $10 \mathrm{~dB}$ offset between the peak of each sinusoidal signal component and the corresponding gammatone filter is applied since it was empirically found to match well with many distorted signals. Next, the effect of the hearing threshold is added by estimating the $F$-weighting function [9] for audio frequencies, together with a user-defined sound pressure level (SPL) value. The net effect of the masking curves and hearing threshold is obtained by taking the maximum value of the filter magnitude responses and the $F$-weighting function for each frequency.

Finally, the DATK creates a residual spectrum by removing the original sine and the nonaliased distortion components, and displays the residual spectrum in the same figure with the auditory spectrum estimate. Since the residual spectrum consists of the aliased signal components (and noise), one can assume that the aliasing artifacts (or noise) are audible, if the residual spectrum exceeds the auditory spectrum estimate in magnitude at any frequency. It should be noted that although there exist more sophisticated techniques (e.g., $[10,11])$ for estimating the auditory spectrum of complex tones, the use of a relatively simple auditory model in this context is enough to characterize the perceptual point of view. Evaluation in detail requires a detailed model compared against a careful formal listening test, which falls beyond the intended scope of the DATK tool.

The DATK aliasing analysis function is invoked by calling

$$
\text { AliasingAnalysis (freq, duration, SPL) }
$$

where freq is the frequency of the sine. The sine frequency should be selected from the normal frequency range defined for the system under measurement. For example, measuring the aliasing of a digital guitar effects device at a frequency of $16 \mathrm{kHz}$ would not yield realistic results, since the electric guitar signal usually has very little energy above $15 \mathrm{kHz}$ [12]. The duration parameter sets the duration of the sine signal 


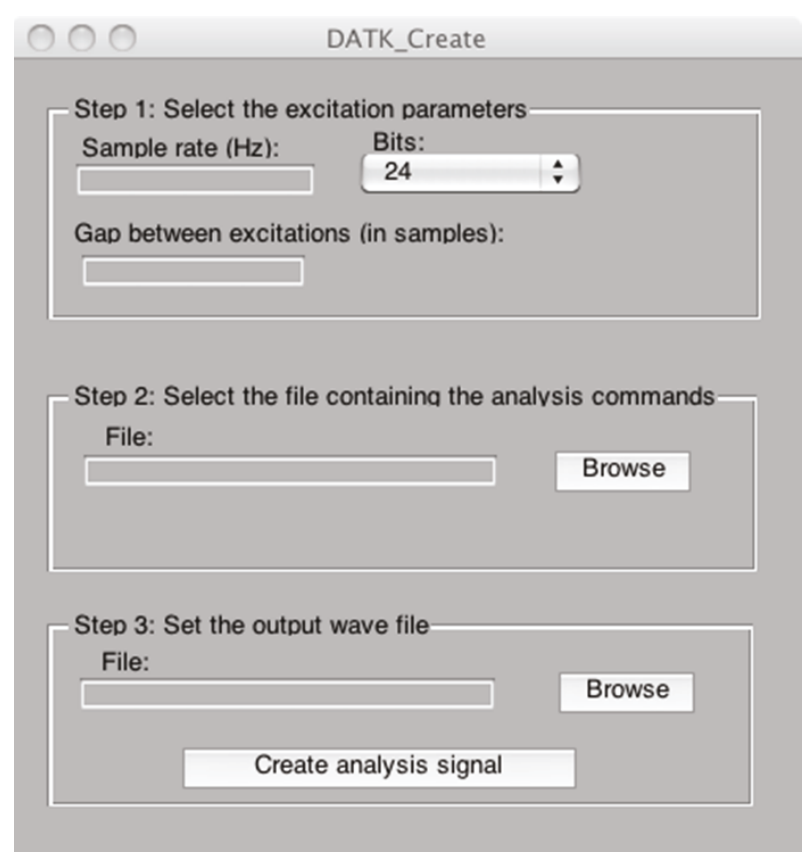

FIgURE 2: Dialog box for creating the analysis signal.

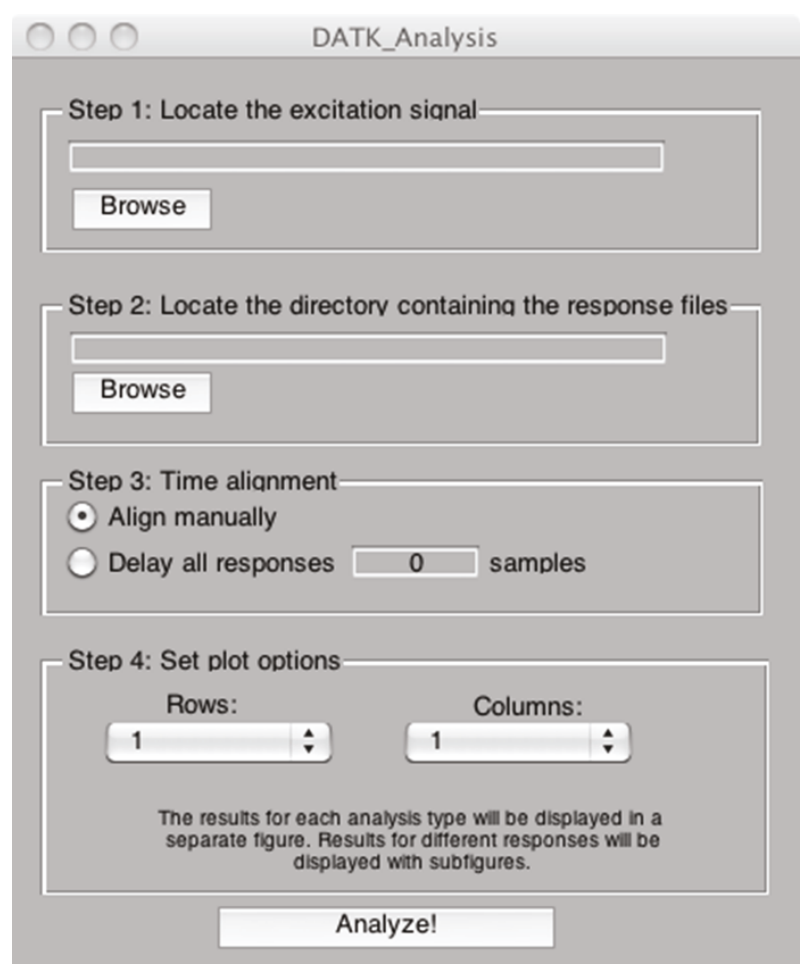

Figure 3: A dialog box for analyzing the system responses.

(in seconds), and the SPL parameter is the estimated Fweighted sound pressure level at which the distorted sine signal would be played.

2.7. Developing Additional Analysis Functions. The user can append the DATK's collection of analysis tools by developing his or her own signal generation and analysis functions. Although the DATK can be operated as a standalone program, developing additional analysis tools requires the use of Matlab. Appending the DATK to include custom userdefined analysis tools is relatively simple, provided that the user is familiar with basic signal processing tasks on Matlab. The user-defined analysis tools should comply with some consistency rules, namely that

(i) there is a function for creating an excitation signal, possibly according to parameters set by the run-time user,

(ii) there is an analysis function which accepts a response signal and plots an analysis figure accordingly,

(iii) any additional parameters that the analysis function requires are generated as metadata by the excitation creation function and stored in a file.

More detailed instructions on developing custom analysis tools for DATK can be found on the DATK website: http:// www.acoustics.hut.fi/publications/papers/DATK/. After developing the custom analysis tools, the user can easily compile a standalone version of the updated DATK using Matlab's deploytool-function.

\section{Case Study: Measurement on the Real and Virtual AC30 Guitar Amplifiers}

This section illustrates the use of the standalone DATK software by measuring the distortion characteristics of an AC30 guitar tube amplifier and two of its virtual analog versions. The main purpose of this section is to provide an example case for producing distortion measurements using the DATK, rather than to perform a detailed interpretation of the operation of the devices. Thus, the emphasis is on explaining the measurement procedure, and what is the useful information in the analysis results, without trying to explain why the amplifier or its virtual versions have a certain effect on the signal.

3.1. VOX AC30. The AC30 is an iconic guitar tube amplifier, first introduced by the VOX company in 1959 [13]. This class $A B$ [14] amplifier uses four cathode-biased EL-84 tubes in the output stage. In 1961, a "Top Boost"-unit, an additional circuit box including treble and bass controls and an extra gain stage was introduced for the AC30. Due to its immediate popularity among users, the Top Boost unit was integrated into the AC30 circuitry from 1963 onwards. The distinct "jangly" sound of the AC30 amplifier can be heard on many records from several bands such as the Beatles, The Rolling Stones, The Who, Queen, R. E. M., and U2, from the last five decades.

A custom-built AC30 amplifier, illustrated in Figure 1, was chosen as a test example for the DATK. This amplifier (referred to as "real AC30" in the following) is a relatively faithful reproduction from the original AC30 circuit schematics, although the tremolo circuit has been omitted. In addition to the real AC30, the distortion measurements 


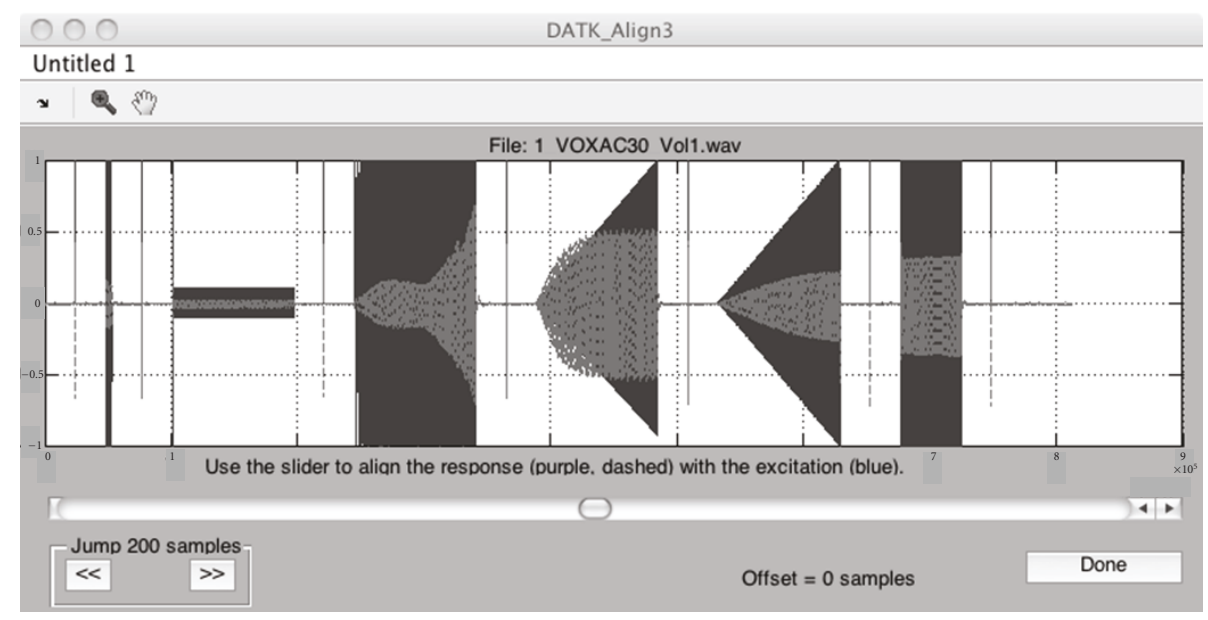

FIGURE 4: Graphical user interface for manual alignment of the analysis signal.

were conducted on two commercial software emulators, namely the Top30 unit of the Overloud TH1 [15] plugin, and the FOX ACS-45 unit of the Peavey ReValver [16] plugin (abbreviated TH1 AC30 and ReValver AC30, resp., in the following).

3.2. Measurement Setup. The distortion behavior of the real AC30 was measured with the DATK software running on a $2.4 \mathrm{GHz}$ MacBook (Intel Core 2 Duo, $4 \mathrm{~GB}$ RAM) laptop computer with M-Audio Firewire 410 as the audio interface. The Reaper software [17] was used in playing the excitation and recording the responses. An $8 \Omega$ power resistor was used as a load for the amplifier. The amplifier output signal was obtained by recording the voltage over the load resistor using a custom-built attenuator/buffer circuit to eliminate the impedance coupling between the amplifier and the audio interface. It should be noted, that although real tube amplifiers usually require a separate attenuator/buffer circuit, many other distortion devices, such as effects pedals, may directly be connected to the audio interface. The highimpedance bright channel was used on the amplifier, and the tone knobs were all set to 12 o'clock. Different responses were measured by varying the "Volume bright" knob, which in practice controls the gain for the bright channel. On the software plugins also, the bright channel was selected (called "Brilliant" on the ReValver AC30), all tone controls were set to 12 o'clock, and different recordings were made while varying the channel gain. It should be noted that since the real AC30 was operated without a loudspeaker, the loudspeaker emulation was switched off for both virtual plugins.

3.3. Creating the Analysis Signal. A complete analysis was performed on the three systems. The five functions were stored in a text file, with the following parameter values:

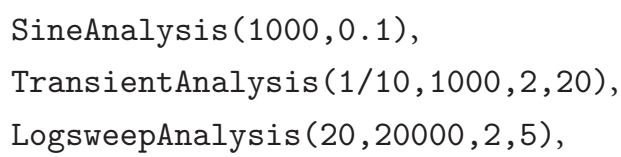

$$
\begin{aligned}
& \text { IMDAnalysis }(6000,1270,1,4,2) \text {, } \\
& \text { AliasingAnalysis }(5000,1,80),
\end{aligned}
$$

This file was read during the analysis by DATK. Next, the excitation signal was created by double-clicking the DATK_Create program icon, which opens a dialog box illustrated in Figure 2. At step 1, a sampling frequency of $48000 \mathrm{~Hz}$ and a bit length equal to 24 were selected from the dialog box. A gap of 24000 samples (corresponding to half a second with the selected sample rate) was chosen between the individual excitations. The purpose of this segment of silence between the measurement signal bursts is to ensure that the different measurements do not affect each other, that is, that the previous response signal has faded before a new one begins. At step 2, the recently created text file was selected using the "Browse"-button, and at step 3, the name and path for the excitation file was selected. Finally, the "Create analysis signal"-button was pressed, resulting in a command-line message denoting that the excitation signal was successfully created.

3.4. Recording. The excitation signal was imported by the recording software, and it was played to the real AC30 while recording its output onto another track. Three recordings were made with different settings for the "volume bright" knob: 9 o'clock (low-gain), 12 o'clock (middle gain), and full (high gain). The three outputs were individually saved as.wav files within the same directory. Next, the TH1 plugin with only the Top30 unit enabled (and the channel gain knob set to 9 o'clock) was added as an effect on the excitation track, and the response was saved as a wave file to the same directory as the real AC30 response files. The TH1 AC30 response recordings were repeated two times with the virtual channel gain knob set to 12 o'clock and full, in order to obtain response wave files with all three gain settings. Finally, the TH1 plugin was replaced with the Peavey ReValver plugin with only the FOX ACS-45 unit enabled, and the same operations (adjust gain, render output wave file, repeat) were carried out three times. Thus in the end, 


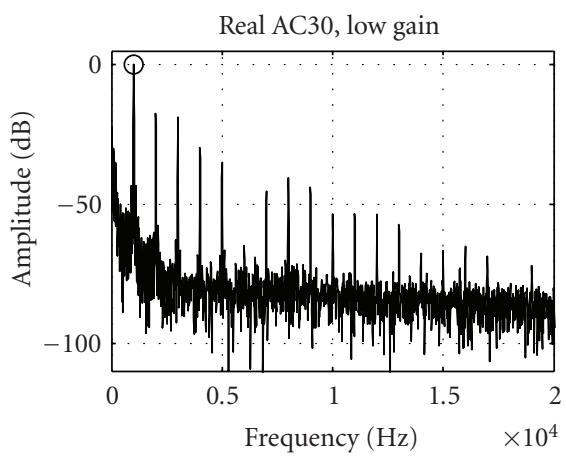

(a)

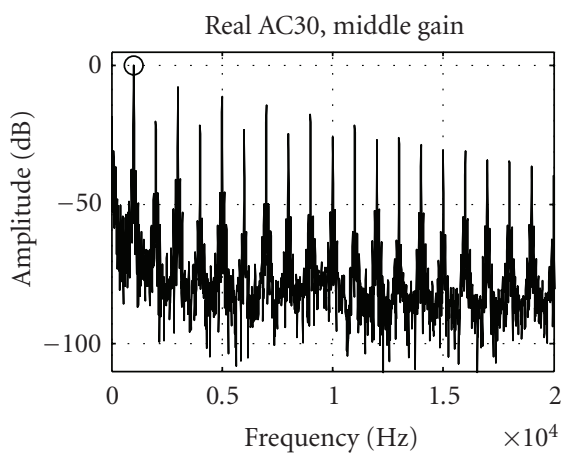

(d)

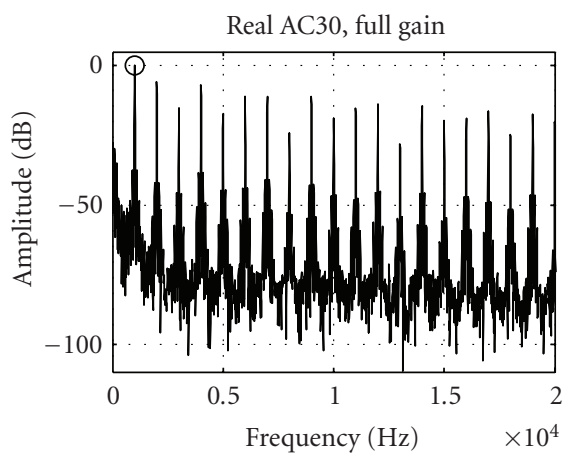

(g)

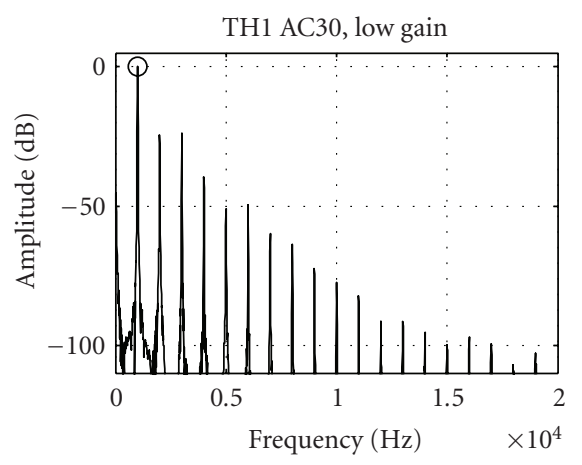

(b)

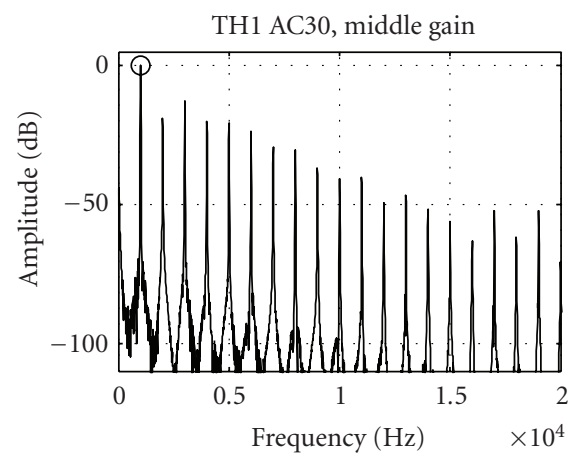

(e)

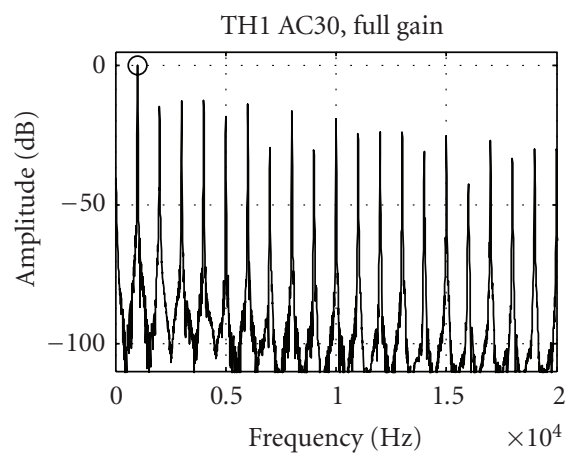

(h)

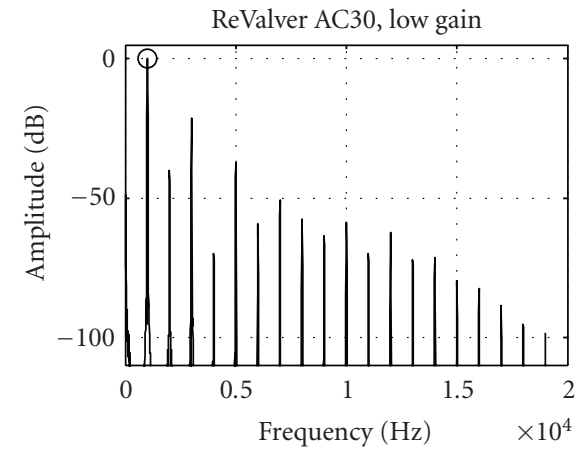

(c)

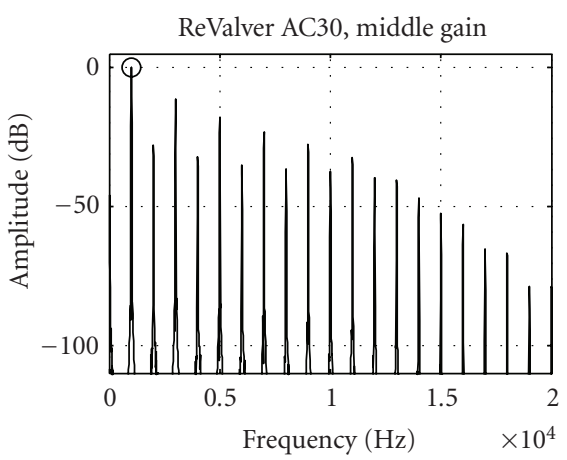

(f)

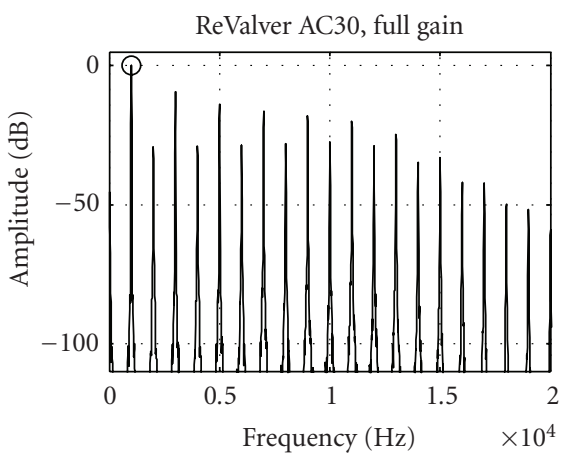

(i)

Figure 5: Response spectra to a $1 \mathrm{kHz}$ sinusoid for the real AC30 (left column), the Top30 unit of the TH1 software plugin (middle column), and the FOX ACS-45 unit of the ReValver software plugin (right column). The user adjustable gain knob was first set to 9 o'clock (top row), then to 12 o'clock (middle row), and finally to full (bottom row). As expected, the real AC30 has a higher noise floor than the digital versions.

the response directory contained 9 response signals as.wav files with sample rate of $48 \mathrm{kHz}$ and 24-bit resolution.

3.5. Signal Analysis. The analysis part of the DATK was started by double-clicking the DATK_Analyze-program icon, which opens a dialog box illustrated in Figure 3. First, the wave file containing the excitation signal and the directory containing the response files were located using the "Browse" buttons at steps 1 and 2 . The next pane, step 3, defines the type of time alignment between the excitation and response signals. The "Align manually" option, which allows the user to individually set the time alignment, was used. The other option allows the user to define a common delay (positive or negative) for all response files. A common delay value may be useful, for example if all responses are obtained from the same device since the signal delay is typically identical between different responses. Since there were three versions of the AC30 with three gain settings each, three rows and three columns were selected at step 4 to display the nine analysis subfigures. Finally, the distortion analysis was started by pressing the "Analyze!" button.

Since manual alignment was chosen at step 3 of the analysis dialog box, the DATK next opens a time alignment window, displayed in Figure 4. Here, the user's task is to move the response signal (denoted with a dashed purple line) in time using a slider, so that it coincides with the excitation signal (denoted with a solid blue line). The resolution of the time alignment is one sample, and the zoom option can be used in adjusting the time offset as carefully as possible. After 


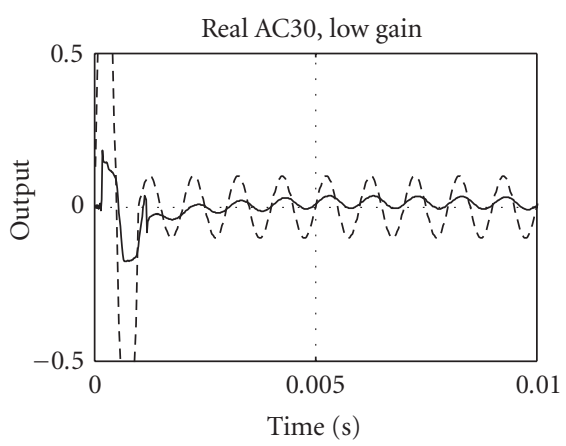

(a)

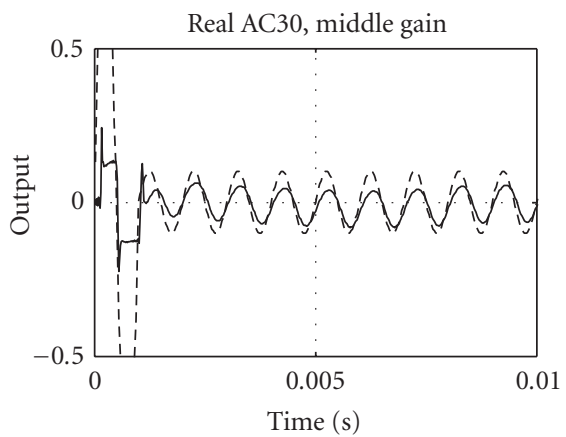

(d)

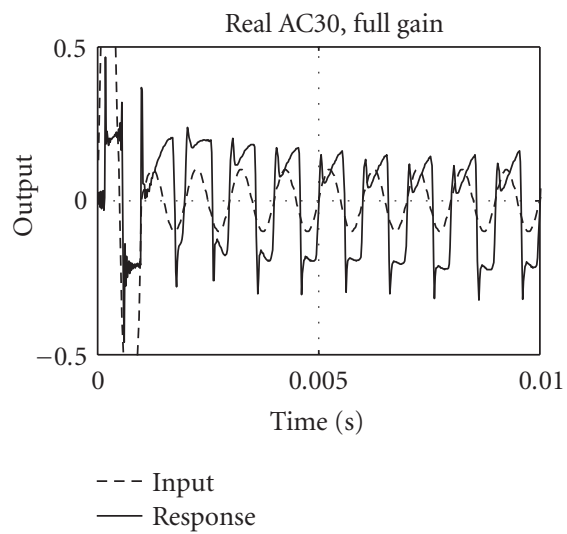

(g)

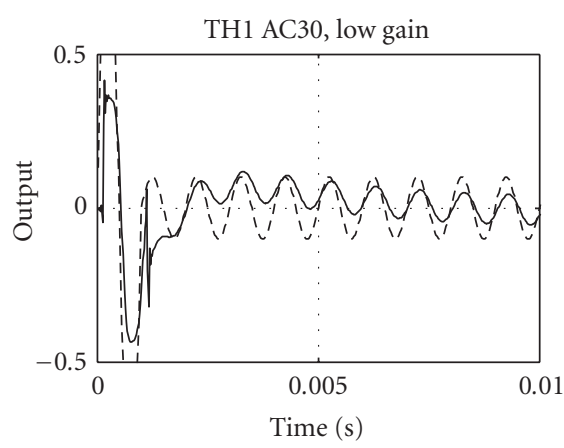

(b)

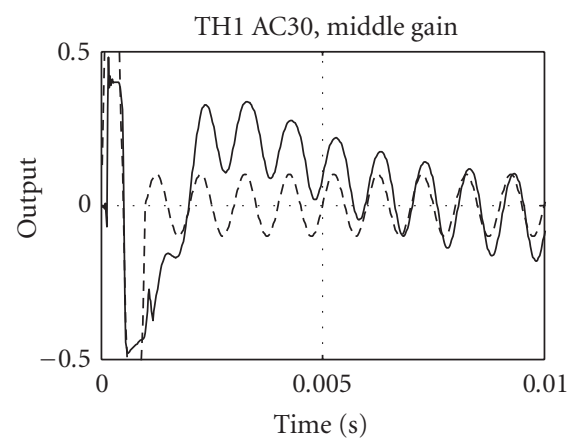

(e)

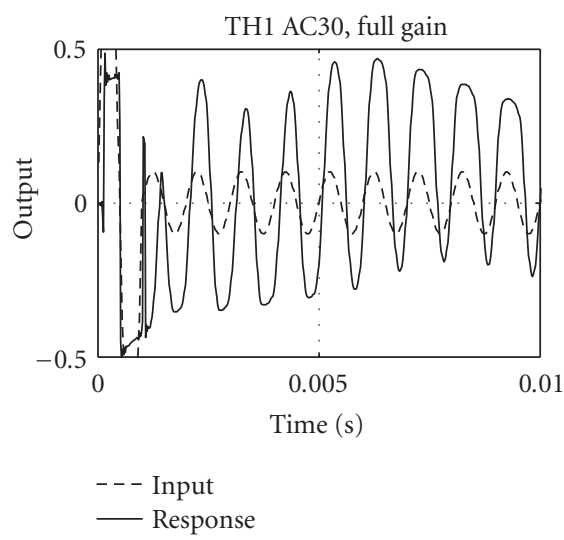

(h)

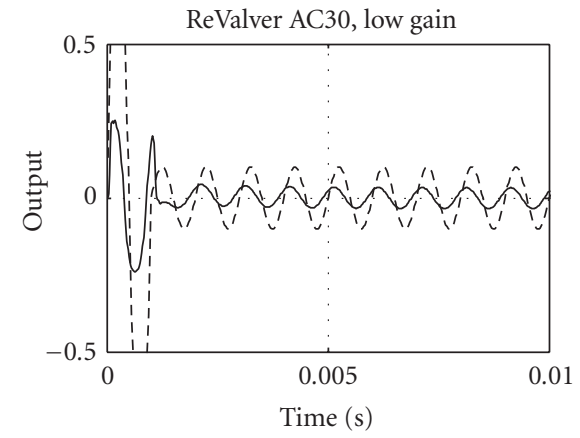

(c)

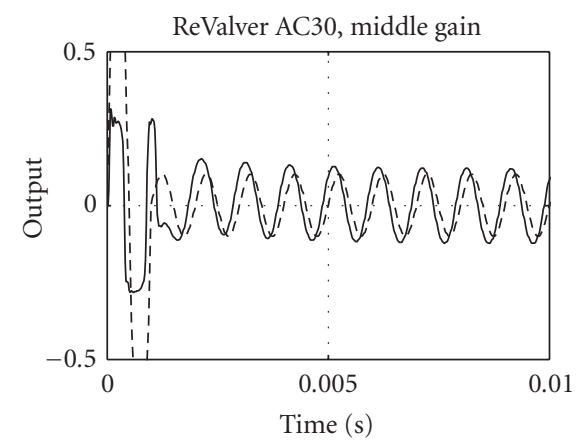

(f)

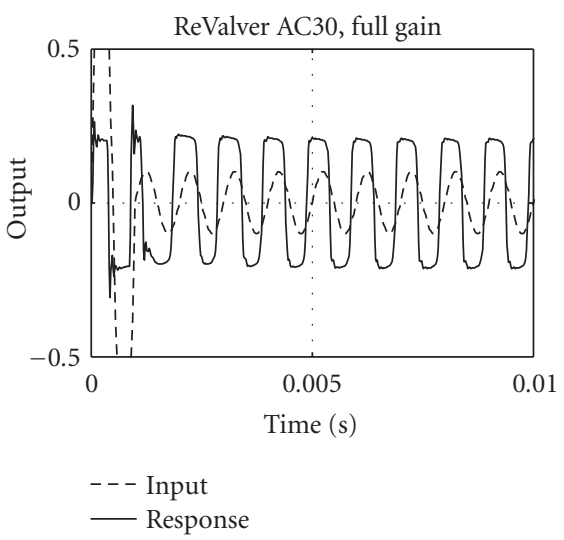

(i)

FIGURE 6: Transient analysis waveforms for the real AC30 (left column), the TH1 AC30 (middle column), and the ReValver AC30 (right column). The input signal is illustrated with a dashed line, while the solid line denotes the measured response. The gain knob was first set to 9 o'clock (top row), then to 12 o'clock (middle row), and finally to full (bottom row).

the user has aligned all the responses with the excitation, the DATK begins the analysis calculation and draws the result figures in the order defined in the analysis command text file.

3.6. Results. Prior to performing the analysis using DATK, the three AC30 variants were tested with an electric guitar. The output signals from all test cases were fed to headphones through a TH1 loudspeaker cabinet simulator in order to obtain a more natural electric guitar sound. This informal and highly subjective playing test revealed that the real AC30 had a certain "smooth" distortion type that was not entirely captured by either software plugins, although the TH1 AC30 managed to get closer to it than the ReValver AC30, which sounded somehow too "harsh", especially when using full gain.

The first analysis plot drawn by the DATK is the sine analysis figure, illustrated in Figure 5. With low-gain settings (top row), all three AC30 variants share a similar lowpasstype spectral envelope. The amplitude ratios of the first few harmonic components on the TH1 AC30 show a relatively good match with the real AC30, while the low-order even harmonics are more suppressed with the ReValver AC30. Interestingly, the 6th harmonic is heavily attenuated in the real AC30 response, while the virtual versions do not mimic this. 


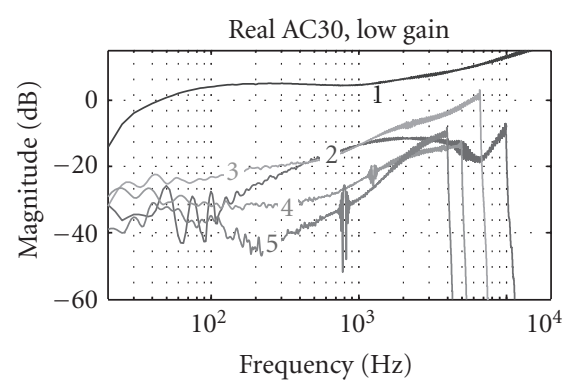

(a)

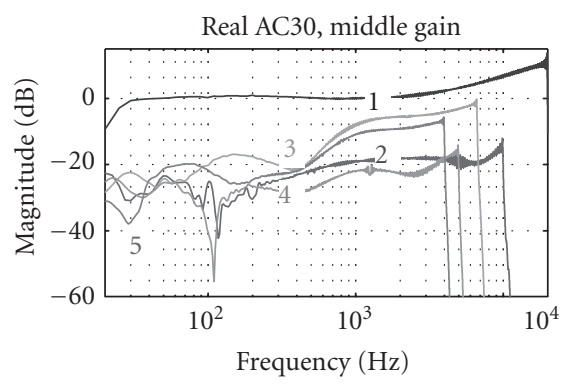

(d)

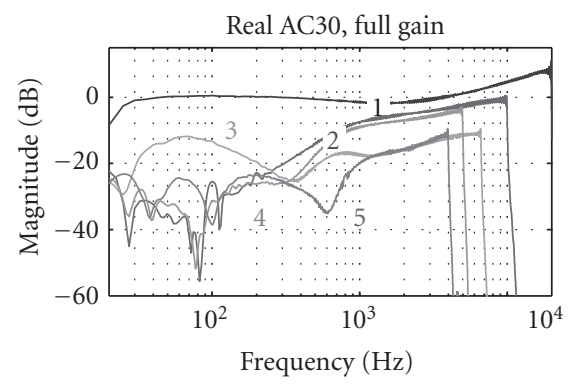

(g)

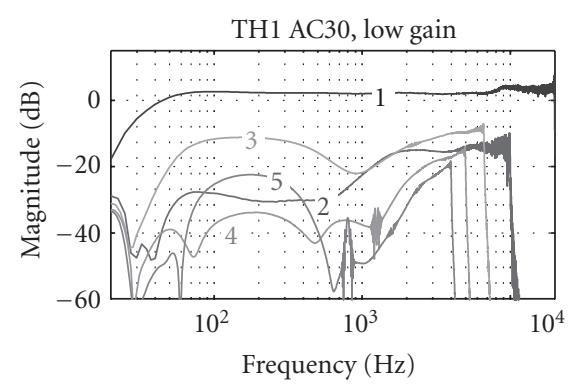

(b)

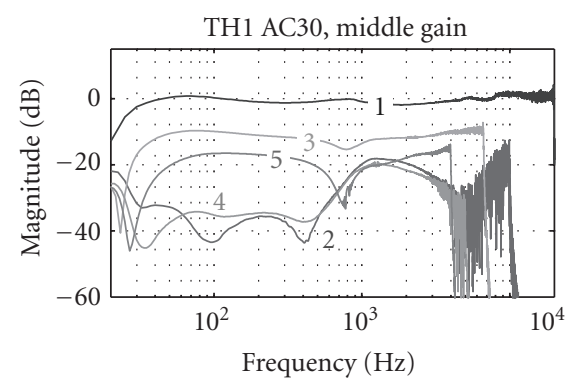

(e)

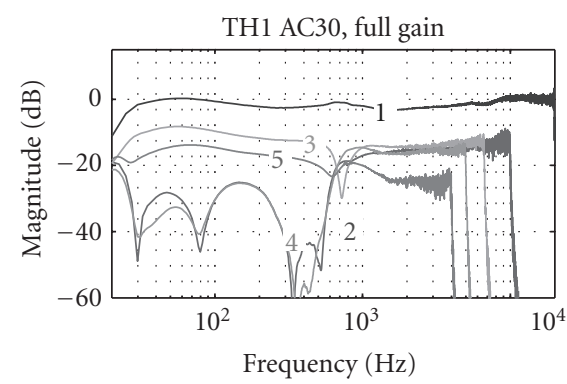

(h)

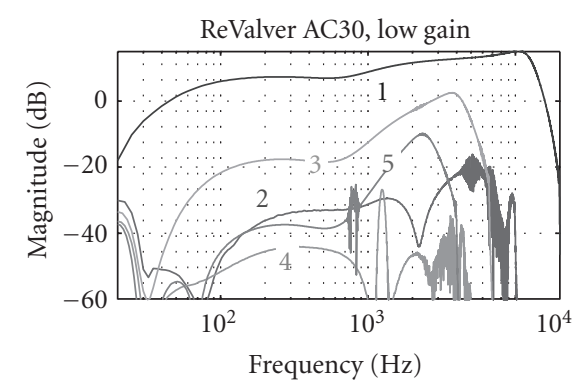

(c)

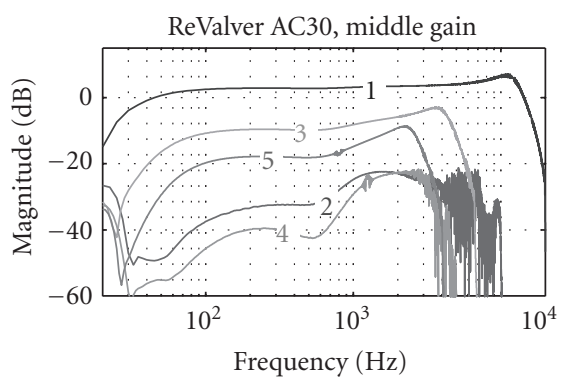

(f)

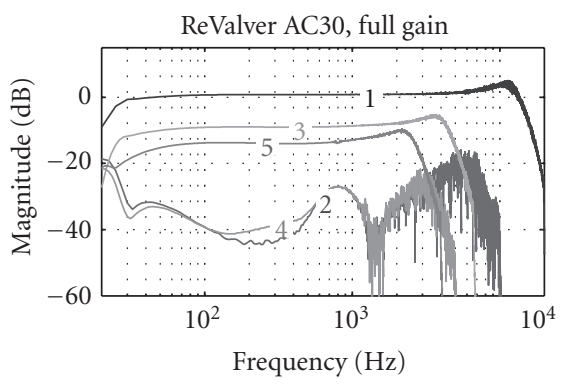

(i)

FIgURE 7: Logsweep analysis of the real AC30 (left column), the TH1 AC30 (middle column), and the ReValver AC30 (right column). The gain knob was first set to 9 o'clock (top row), then to 12 o'clock (middle row), and finally to full (bottom row). The numbered curves represent the order of the distortion, so that curve 1 corresponds to the linear magnitude response, curve 2 to the 2 nd order harmonic distortion, curve 3 to the $3 \mathrm{rd}$ order harmonic distortion, and so forth. The 6th order and higher distortion components are not illustrated.

Increasing the gain level to medium (middle row), the even harmonic components become more suppressed in the real AC30 response, while the overall spectral envelope gets brighter. The ReValver AC30 response seems to simulate this even-order distortion component attenuation well. Both virtual plugins show a slightly stronger lowpass-effect than the real AC30 with medium gain. For full gain settings, the even-order distortion components exceed the odd-order components in the real AC30 response. For the ReValver AC30, the opposite happens: the odd-order components are significantly louder. As expected, the overall spectral envelope gets even brighter with increased gain in all three AC30 variants. Looking at all responses in Figure 5, it seems that the spectrum of the real AC30 response varies strongly on the gain level and this change is more subtle with the software versions.

The second analysis type produces the transient response plot, illustrated in Figure 6, where the dashed line denotes the input signal, while the response waveform is drawn with a solid line. The vertical axis has been zoomed in between $-0.5-0.5$ to better illustrate the dynamic effects. It can be seen in the figure that the initial transient is hardclipped in nearly all cases. For low-gain settings (top row), the real AC30 shows a slight bias shift after the transient, without a strong effect on the waveform. The TH1 AC30 response experiences a slightly stronger bias shift, with a heavier distortion immediately after the transient, while the ReValver AC30 response is more static. For medium and full gain settings (middle and bottom row), the real AC30 shows a slight attenuation right after the transient, which might indicate blocking distortion [7]. The TH1 AC30 response shows an exaggerated bias shift due to the transient, while the ReValver AC30 response is again more static. With full gain, the real AC30 waveform is more severely distorted than with the software plugins.

The third analysis figure is the logsweep response plot, illustrated in Figure 7. Here, it can be seen that the linear response (curve 1) of the ReValver AC30 shows a similar 


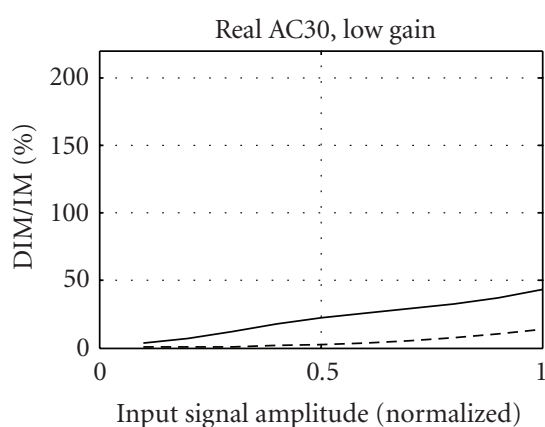

(a)

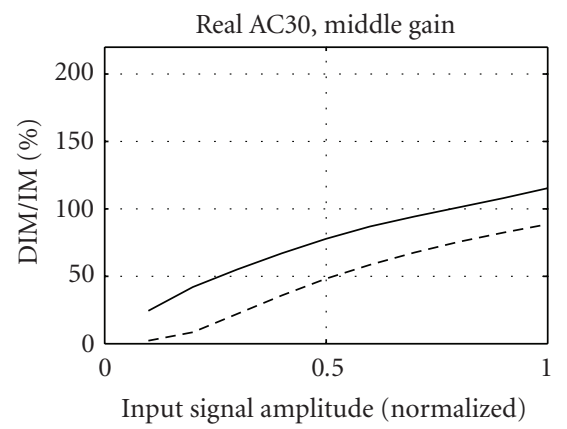

(d)

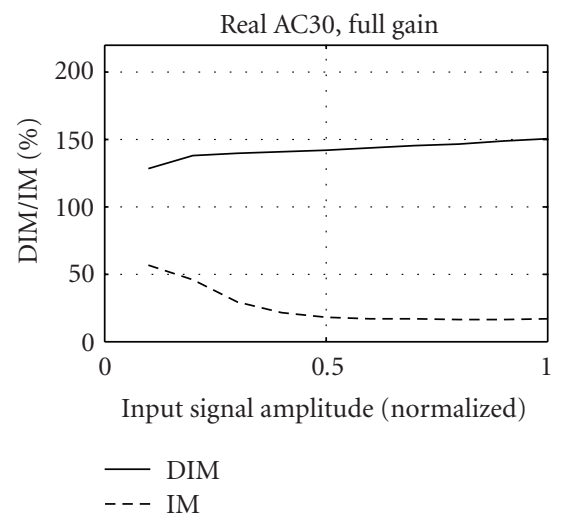

(g)

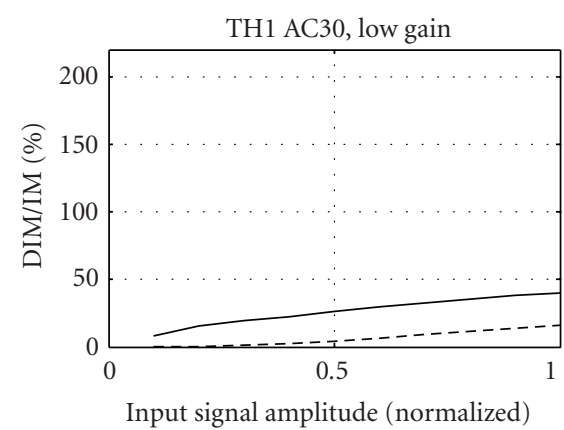

(b)

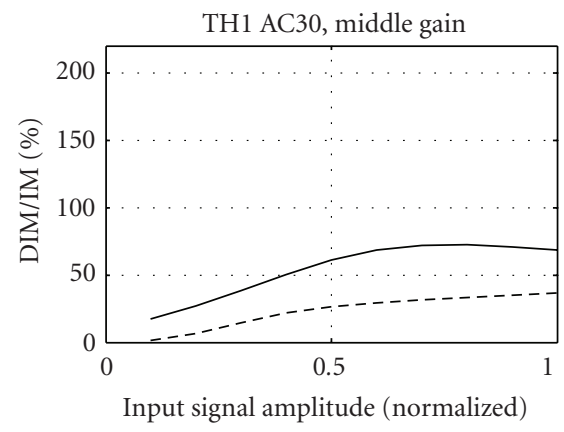

(e)

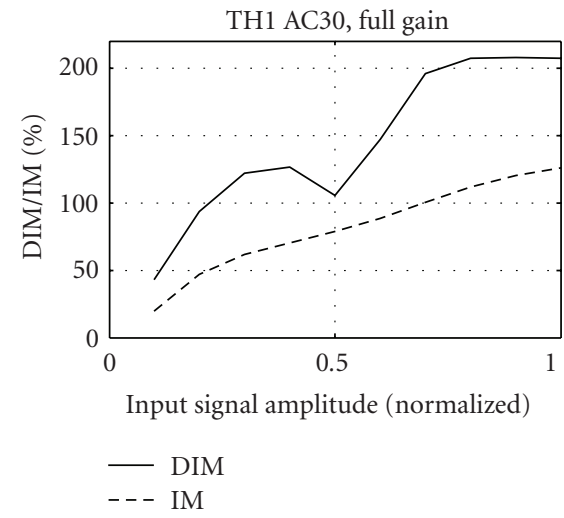

(h)

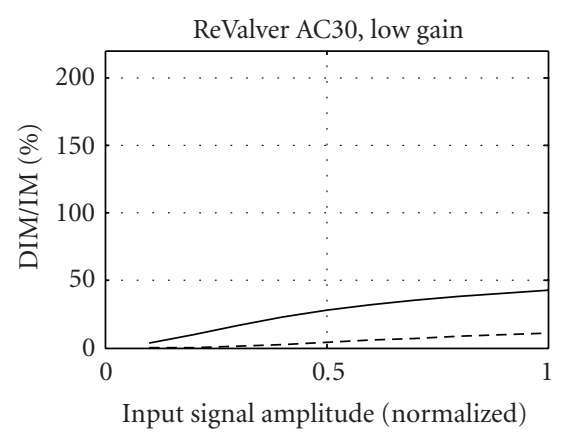

(c)

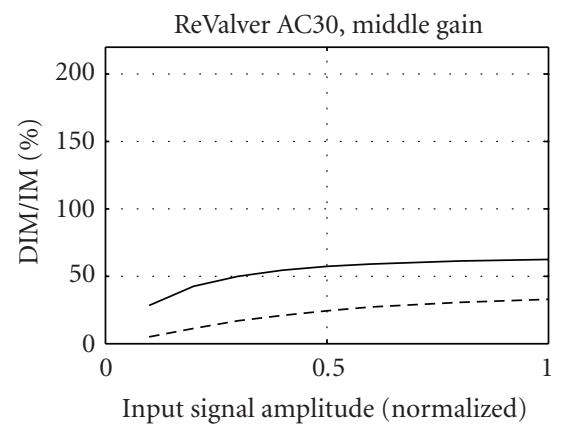

(f)

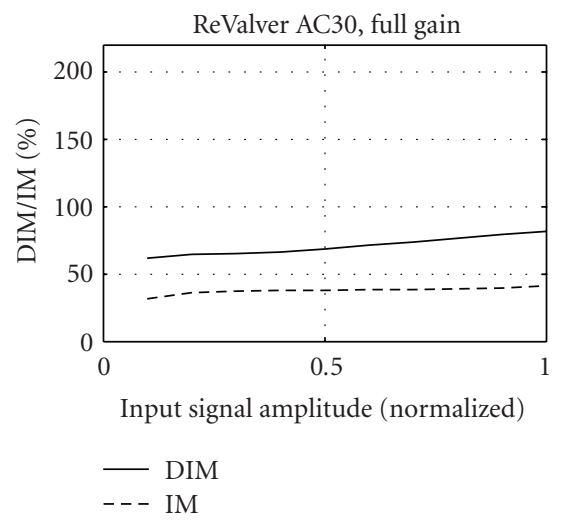

(i)

FIGURE 8: Intermodulation distortion analysis of the real AC30 (left column), the TH1 AC30 (middle column), and the ReValver AC30 (right column). The gain knob was first set to 9 o'clock (top row), then to 12 o'clock (middle row), and finally to full (bottom row). The solid line illustrates the amount of dynamic intermodulation distortion, while the dashed line denotes the amount of static intermodulation distortion, both as a function of the normalized input signal level.

highpass behavior in the audio range as the real AC30, while the TH1 AC30 response is flatter. For low and medium-gain settings, the distortion components on the real AC30 have a highpass nature. The even harmonics on the TH1 AC30 mimic this somewhat, while the odd harmonics are more pronounced in the low frequencies than with the real AC30. The odd harmonics on the ReValver AC30 have a highpass trend for low and medium-gain, but the even harmonics are attenuated more than in the real AC30. For full gain, the second and third harmonics on the real AC30 are very strong at high frequencies. Neither software plugins have such a high distortion in the kilohertz range as the real AC30. Furthermore, neither plugins mimic the boosting of the third harmonic and simultaneous suppression of the fifth harmonic at low frequencies (near $70 \mathrm{~Hz}$ ).

The fourth analysis result created by the DATK is the intermodulation distortion analysis plot, illustrated in Figure 8. It shows the dynamic intermodulation (DIM) distortion percentage with the solid line, and the static intermodulation (IM) distortion percentage with a dashed line, both as a function of the normalized input signal amplitude. As can be seen in the figure, the DIM distortion exceeds the static IM distortion in all cases. For low- and medium gain settings (top and middle row), the DIM/IM distortion increases monotonically with input signal amplitude in nearly the same way for all three AC30 variants. 


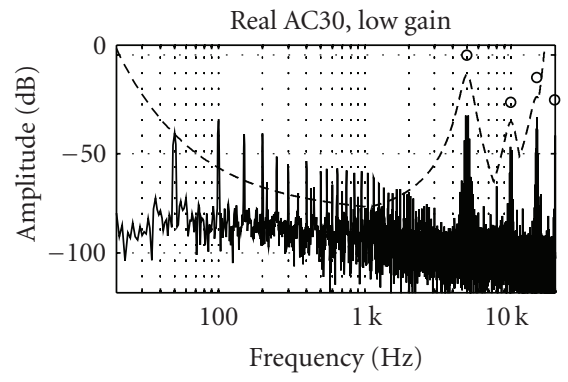

(a)

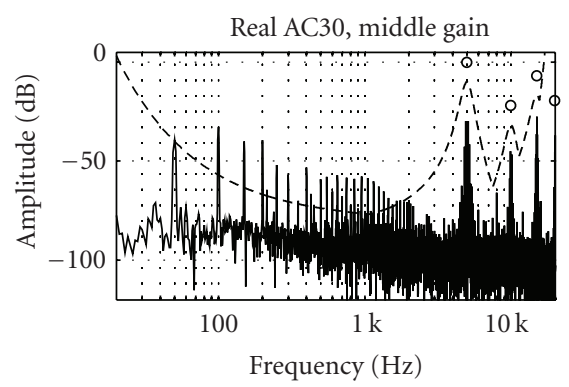

(d)

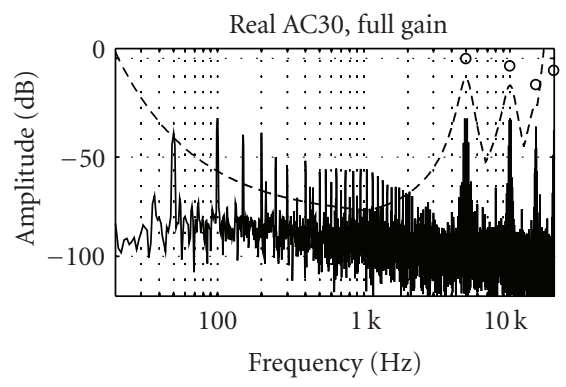

$\begin{array}{cl}\text { - Signal } \\ --- & \text { Aud. spec. } \\ - & \text { Aliasing }\end{array}$

(g)

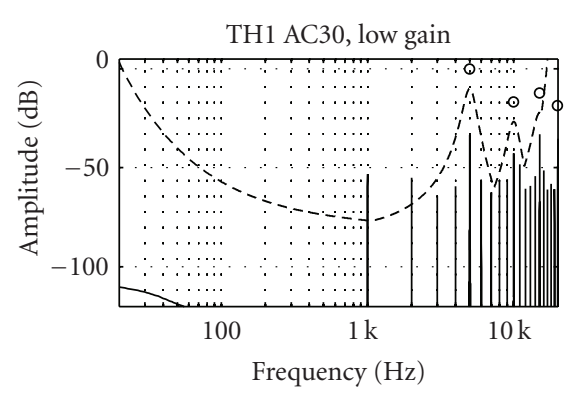

(b)

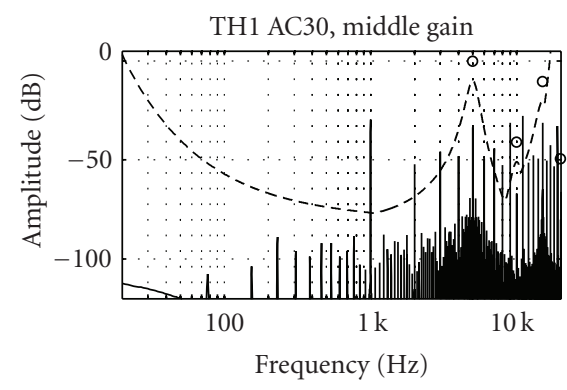

(e)
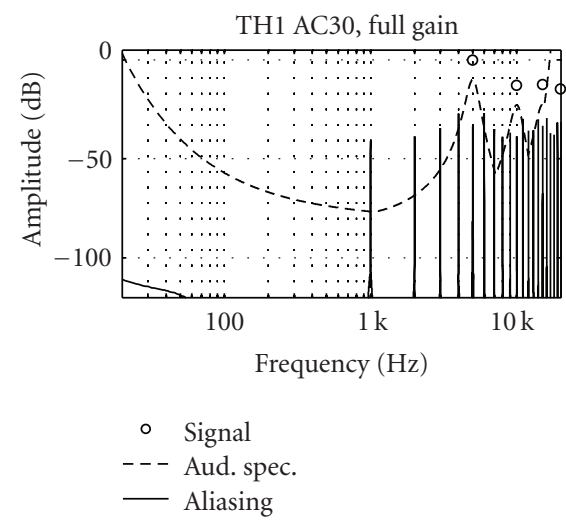

(h)

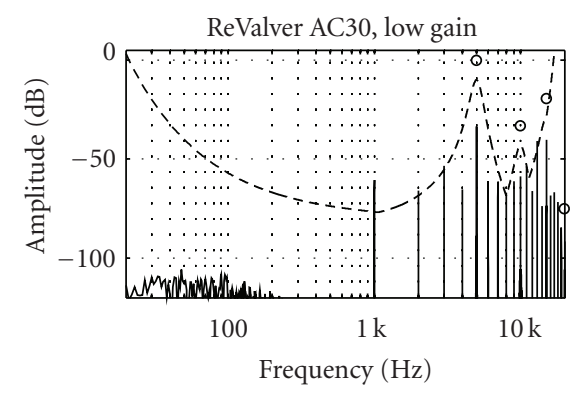

(c)

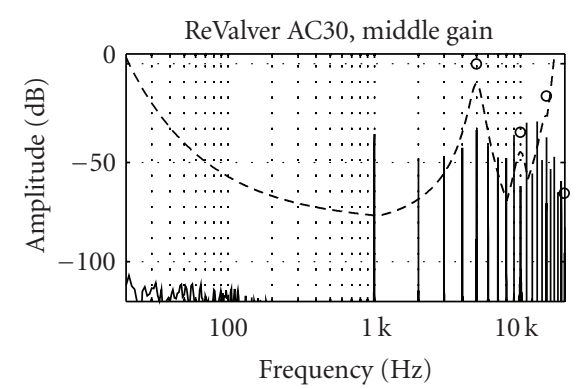

(f)
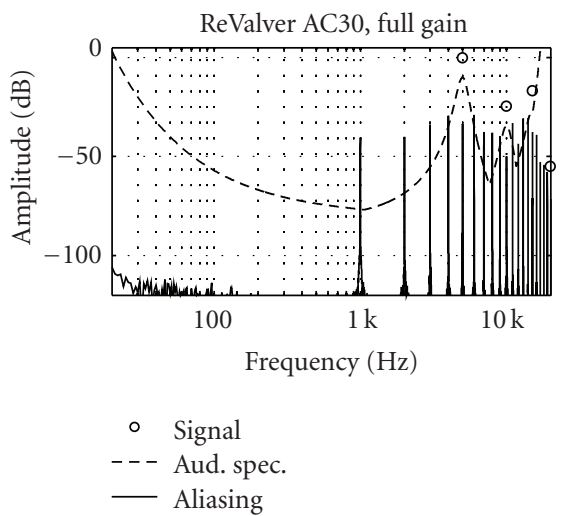

(i)

FIGURE 9: Aliasing analysis for the real AC30 (left column), the TH1 AC30 (middle column), and the ReValver AC30 (right column), when a $5 \mathrm{kHz}$ sine is used as input. The gain knob was first set to 9 o' clock (top row), then to 12 o'clock (middle row), and finally to full (bottom row). In the figures, the circles denote the peaks of the harmonic components, while the solid line stands for aliased signal components and noise. The dashed line illustrates an auditory spectrum estimate. In both software plugins (middle and right column), the aliased components greatly exceed the estimated auditory threshold, thus suggesting that the aliasing is clearly audible. For the real AC30 (left column), the figures show that the $50 \mathrm{~Hz}$ power supply hum and its harmonics are also audible.

With full gain (bottom row), however, the intermodulation distortion figures reveal stronger differences. On the real AC30, the static IM distortion decreases with increasing input signal amplitude, while the DIM distortion remains nearly constant. The TH1 AC30 shows an interesting notch in the DIM distortion curve, indicating that there could be a local minimum of DIM distortion with input signals with a certain amplitude. The DIM/IM distortion on the ReValver AC30 with full gain seems to be relatively low and largely unaffected by the input signal amplitude.

In general, it seems that there are no large differences in the intermodulation distortion behavior between the real and simulated AC30 variants at low- and medium gain settings. However, the underlying mechanisms causing the different full-gain intermodulation distortion behavior between the AC30 variants should be examined in detail in further studies.

The fifth and final analysis plot is the aliasing analysis, depicted in Figure 9. It illustrates the estimated auditory spectrum (dashed line) caused by a distorted $5 \mathrm{kHz}$ sine signal. The amplitude peaks of the distorted signal are plotted with circles. The solid lines in the figures illustrate the aliased signal components and measurement noise.

As can be seen in Figure 9, the real AC30 (first column) has a relatively high noise level due to the $50 \mathrm{~Hz}$ power supply hum and its harmonic components. This power supply 
noise is clearly audible also in quiet parts when using the amplifier in a real playing situation. Interestingly, the aliasing analysis plots for both virtual AC30 plugins (second and third column) show severe aliasing phenomenon. The aliased component at $1 \mathrm{kHz}$ is roughly $40 \mathrm{~dB}$ above the auditory spectrum estimate at full gain settings, making it clearly audible. In fact, the heavy aliasing behavior can strongly be heard also by listening to the logsweep responses of the AC30 plugins. It should be noted that the power supply noise is generally less irritating than aliasing noise, since the former stays largely constant regardless of the input signal. Aliasing noise, in turn, changes radically according to the input signal, and can it be an especially undesired phenomenon when playing high bent notes, since when the original tone moves up in frequency, the strongest aliased components move down, creating an unpleasant effect.

After all the analysis figures have been created, the DATK analysis part is finished. For the $2.4 \mathrm{GHz}$ MacBook test computer, it took 76 seconds to analyze the AC30 responses (66 seconds when running under Matlab), while clearly most of the time is spent in performing the logsweep analysis.

\section{Conclusion}

A new software tool for performing rapid analysis measurements on nonlinearly distorting audio effects was presented. The software is called Distortion Analysis Toolkit (DATK), and it is freely available for download at http://www .acoustics.hut.fi/publications/papers/DATK/. The software operates by first creating an excitation signal as a.wav file according to the specifications set by the user. Next, the user feeds this excitation signal as an input to a physical or virtual distorting audio effect and records the output. Several recordings can be made if the distorting effect's control parameter variations are to be analyzed. Finally, the introduced software analyzes the response files and displays the analysis results with figures. The software is developed in Matlab language, but it can also be operated in standalone mode. The DATK includes five distortion analysis methods, and additional analysis techniques can be appended to it using Matlab.

The usage of the DATK was illustrated with a case study, where a custom-built AC30 guitar tube amplifier and two commercial software simulations, TH1 AC30 and ReValver AC30, were measured and compared. The amount and type of distortion on the real AC30 were found to be strongly dependent on the channel gain, as well as input signal frequency. Especially the amplitude ratios between the first few harmonic components show a complex nonmonotonous dependency from the channel gain. Although both software plugins successfully simulate the overall distortion characteristics in a broad sense, their response is more static than the real AC30 response. Waveform responses to a transient input signal reveal that the nonlinearity in the real AC30 is dynamic. Also the tested TH1 AC30 plugin shows strong dynamic behavior, while the ReValver AC30 plugin is more static. Intermodulation distortion analysis did not reveal any major differences between the three AC30 variants for low- and medium-gain settings, although some differences could be observed under full gain operation. Clearly audible aliasing noise was observed for both software plugins with sinusoid inputs when running a $48 \mathrm{kHz}$ sample rate, whereas the real AC30 suffered from power supply hum. In a practical guitar playing condition, the aliasing noise on the tested software plugins is in most cases negligible due to the complex spectrum of the guitar. High bent notes can, however, reveal audible aliasing artifacts even when operating at a $96 \mathrm{kHz}$ sample rate.

\section{Acknowledgments}

This research is funded by the Aalto University. The author is grateful to Vesa Välimäki for helpful comments. Special thanks are due to Ari Viitala for building the AC30 amplifier.

\section{References}

[1] J. Pakarinen and D. T. Yeh, "A review of digital techniques for modeling vacuum-tube guitar amplifiers," Computer Music Journal, vol. 33, no. 2, pp. 85-100, 2009.

[2] A. Farina, "Simultaneous measurement of impulse response and distortion with a swept-sine technique," in Proceedings of the 108th AES Convention, Paris, France, February 2000, preprint 5093.

[3] P. D. Hatziantoniou and J. N. Mourjopoulos, "Generalized fractional-octave smoothing of audio and acoustic responses," Journal of the Audio Engineering Society, vol. 48, no. 4, pp. 259280, 2000.

[4] M. Otala, "Transient distortion in transistorized audio power amplifiers," IEEE Transactions on Audio and Electroacoustics, vol. 18, no. 3, pp. 234-239, 1970.

[5] E. Leinonen, M. Otala, and J. Curl, "A method for measuring transient intermodulation distortion (TIM)," Journal of the Audio Engineering Society, vol. 25, no. 4, pp. 170-177, 1977.

[6] V. Välimäki and A. Huovilainen, "Oscillator and filter algorithms for virtual analog synthesis," Computer Music Journal, vol. 30, no. 2, pp. 19-31, 2006.

[7] J. Pakarinen and M. Karjalainen, "Enhanced wave digital triode model for real-time tube amplifier emulation," IEEE Transactions on Audio, Speech and Language Processing, vol. 18, no. 4, pp. 738-746, 2010.

[8] R. D. Patterson, "The sound of a sinusoid: spectral models," Journal of the Acoustical Society of America, vol. 96, no. 3, pp. 1409-1418, 1994.

[9] R. A. Wannamaker, "Psychoacoustically optimal noise shaping," Journal of the Audio Engineering Society, vol. 40, no. 7-8, pp. 611-620, 1992.

[10] K. Brandenburg, G. Stoll, Y. F. Dehéry, J. D. Johnston, L. V. D. Kerkhof, and E. F. Schröder, "The ISO-MPEG-1 audio: a generic standard for coding of high-quality digital audio," Journal of the Audio Engineering Society, vol. 42, no. 10, pp. 780-792, 1994.

[11] S. Van De Par, A. Kohlrausch, G. Charestan, and R. Heusdens, "A new psychoacoustical masking model for audio coding applications," in Proceedings of the IEEE International Conference on Acoustic, Speech, and Signal Processing, vol. 2, pp. 18051808, Orlando, Fla, USA, May 2002. 
[12] S. Errede, "Measurement of the electromagnetic properties of electric guitar pickups," Tech. Rep., February 2010, http:// online.physics.uiuc.edu/courses/phys498pom/lab_handouts/ electric_guitar_pickup_measurements.pdf.

[13] J. Elyea, "The history of Vox," April 2010, http://www .voxamps.com/history.

[14] R. Aiken, "Is the Vox AC-30 really class A?" 2002, http://www .aikenamps.com/VoxAC30classA_2.html.

[15] “Overloud TH1,” February 2010, http://www.overloud.com/.

[16] "Peavey ReValver," February 2010, http://www.peavey.com/ products/revalver/index.cfm.

[17] "Cockos Reaper," February 2010, http://www.cockos.com/ reaper. 